\title{
La Gestión Administrativa Municipal. Una aproximación Conceptual
}

Municipal Administrative Management. A Conceptual Approach

\section{Luna Sono, Jaime}

Maestro en Gestión Pública y Gobernabilidad

Universidad César Vallejo - jalunal@ucvvirtual.edu.pe

Correo: jaimelunasono1@gmail.com - ORCID (0000-0001-7374-8149),

https://scholar.google.es/scholar?hl=es\&as_sdt=0\%2C5\&q=jaime+luna+sono\&btnG=

\section{Manuel Jesús Luna Hernández}

Maestro en Gestión Ambiental, Universidad Mayor de San Marcos, Lima-Perú. mesuslunah@yahoo.com,ORCID: 0000-0001-7487-8543

httpsscholar.google.com/citations?user=R7fjbncAAAAJ

Revista Iberoamericana de la Educación

Vol - Especial 12021

e-ISSN: 2737-632x

\begin{abstract}
La Gestión Administrativa Municipal, es hoy un factor clave en el desarrollo de las instituciones de gobiernos locales, y su estudio en profundidad, diagnóstico y mejoramiento incide de manera directa en el denominado espíritu del desenvolvimiento de las actividades administrativas internas de las municipalidades; como objetivo, por lo anterior en el presente documento, se realiza una aproximación conceptual que ayuda a evidenciar la importancia de la gestión administrativa municipal. De igual forma se exponen las diferentes herramientas y métodos, examinándose distintos artículos como de la autora argentina María, Raffino (2020) "Concepto de gestión en la administración", que se pueden emplear para su medición y diagnóstico, que consideran numerosas variables que giran en torno a los valores de la gestión administrativa en el ámbito municipal, obteniéndose como resultados, las creencias, costumbres y prácticas de gestiones municipales, concluyéndose en no sólo la conceptualización, sino esclarecer las causas de la satisfacción e insatisfacción en los usuarios y su incidencia, en los cambios de gestión administrativa municipal.
\end{abstract}


Palabras Claves: Gestion Administrativa Municipal, Usuario.

\begin{abstract}
:
Municipal Administrative Management is today a key factor in the development of local government institutions, and its in-depth study, diagnosis and improvement directly affects the so-called spirit of development of the internal administrative activities of municipalities; As an objective, due to the foregoing in this document, a conceptual approach is made that helps to demonstrate the importance of municipal administrative management. In the same way, the different tools and methods are exposed, which can be used for their measurement and diagnosis and that consider numerous variables that revolve around the values of municipal administrative management, obtaining as results, the beliefs, customs and management practices municipalities, concluding in not only the conceptualization, but also to clarify the causes of satisfaction and dissatisfaction in users and their incidence, in the changes of municipal administrative management.
\end{abstract}

Keywords: Management Administrative Municipal, Users.

\title{
INTRODUCTION
}

Mediante este estudio, se alcanzará el tratamiento y análisis de la Gestión Administrativa, como principal actividad en los gobiernos gubernamentales, en su tercer nivel; considerándose además su gran importancia; debido a que resulta ineludible, que la gestión administrativa en las instituciones municipales, como es en el caso del Perú; estos mecanismos o sistemas, se encuentran creados, dentro de un marco constitucional y político; y comulgan 
como filosofía, la del servicio al ciudadano, así como el bienestar social de ellos. Asimismo, para el estudio de la gestión administrativa, se hace necesario identificar los diferentes elementos que constituyen el concepto:

De acuerdo a lo señalado por Chiavenato (2014), el origen de la gestión administrativa, se inicia desde las remotas épocas, en que los principios elementales se direccionaron a resolver problemas de los grupos de trabajo, por las personas. Se ha establecido hitos prehistóricos, consistentes en las construcciones de la antigüedad, como la de Egipto, Mesopotamia y Asiria. Estos colosos son o vienen a ser los testimonios irrefutables, de las épocas remotas donde existieron hombres, capaces de planear y dirigir los esfuerzos de miles de trabajadores, que lograron construir dichos hitos como las pirámides de Egipto. Ahora bien, a esto debemos sumar los papiros egipcios (1300 a.C.), con lo cual se demuestra la importancia de la administración y organización en la burocracia pública de Egipto. Es en China, donde sostienen prácticas para la buena marcha administrativa en la función pública, como las parábolas de Confucio. Es por eso que Mochón, Mochón y Sáez (2014), comentan que, a través de la historia de las teorías, se entiende que las teorías y prácticas, actuales, tienen marcadas fortalezas y debilidades. De la palabra Gestión Administrativa, se define Gestión, como vocablo proveniente del latín, siendo sus acepciones, la de administrar, dirección, gestión, y/o actividad calificada, en busca de objetivos y medios profesionales. También se sostiene que gestión, correspondería al manejo de recursos de las organizaciones; privadas y estatales o públicas.

Rodríguez, (2010) señala a la gestión: (p. 20), que este proviene del vocablo Management, proveniente de un origen anglosajón; utilizado universalmente por los dedicados a las empresas. lenguaje que traducido al español, se 
entiende como gestión, dirección, administración, gerencia; cuyos conceptos, se debe considerar como sinónimos; en el contexto de la administración; y como funciones básicas de todo lo que es dirección, ligadas a la gestión.

También, Campos y Loza, (2011) sosteniéndose en el diccionario enciclopédico "Gran Plaza y Jaime Ilustrado", puntualiza, a la gestión como la acción y efecto de gestionar o de administrar; que viene a ser ganar, hacer diligencias conducentes para lograr algún negocio u otro. Es así que, en otra concepción, similar especifican a la gestión, el conjunto de acciones de dirección y administración, en una empresa. (p.14).

Estos mismos investigadores, Campos y Loza (2011), subrayan (p. 20): su función básica, según Chiavenato y Fayol; seccionan las empresas en seis grupos, con funciones generales, o sea, técnicas comerciales, financieras, de seguridad, contables y administrativas, (Chiavenato, 1989, p. 79). dirección y administración de una empresa. (Escudero R., 2011), (p.40).

Según, Mochón et al. (2014), señala como enfoque clásico, al conjunto de estudios caracterizado para procurar la eficiencia, detallando, que los trabajadores, sean productivos, y las organizaciones, funcionen con racionalidad. El factor clave, que procuró el progreso de este enfoque, fue el proceso de mecanización, que se inicia, con la Revolución Industrial; en los siglos XVIII y XIX, cuando Inglaterra, posteriormente el resto de Europa continental seguida de Norteamérica; sufrieron transformaciones socioeconómicas, tecnológicas y culturales, es en este momento que aparecen las grandes fábricas, seguido de inmensa producción y mecanización del trabajo. Por lo tanto, la sustitución de las máquinas por la fuerza de trabajo 
humano; dio inicio el abandono del trabajo en los hogares, dando lugar a la producción, en casi totalidad en las fábricas.

Dentro de este contexto y/o enfoque, se destacó la teoría de la administración científica y la teoría general de la administración, como primer enfoque de la teoría de la administración científica y fue el primer enfoque de la administración y con su exponente Frederick W. Taylor, por los años (18561915) (Taylor, 1967), quien sostuvo con mucho esmero, el método científico; para establecer la mejor forma de realizar cualquier actividad en la organización y recomendó la especialización y la división del trabajo. Es así, como hasta la fecha, permanece como base, en el diseño de puestos de trabajo.

Sus ideas originales, sostenían que se debe aplicar el método científico al trabajo de la persona; y en cada acción laboral, lograr la cooperación entre directivos y trabajadores; estableciéndose la clara división del trabajo entre los trabajadores y la dirección; lográndose esto, con una remuneración se considere como premio por los resultados obtenidos, estimulando los incentivos monetarios, a la mejor productividad.

Asimismo, señala que la teoría general de la administración, estudia además a las organizaciones, especialmente a lo que hacen los gerentes; esta teoría destaca la importancia de crear una estructura organizativa que propicie la efectividad y eficiencia en las organizaciones. Los estudios más relevantes fueron la de Fayol (1841- 1925) (Fayol, 1949) y del profesor alemán Max Weber (1864-1920) (Weber, 1947) que esbozaron los principios de la administración. 
Fayol, como ingeniero y economista francés, fue considerado el padre de la estructura funcional de la empresa; elaborando 14 principios, como división del trabajo, autoridad y responsabilidad, disciplina, unidad de mando, unidad de dirección, subordinación del interés particular al interés general, remuneración, centralización, escalafón, orden, equidad, estabilidad en los puestos de trabajo, iniciativa y espíritu de equipo.

En cambio, Weber, sociólogo, filósofo, historiador, jurista alemán, esbozó los principios de la burocracia (teorías de estructuras de autoridad y relaciones basadas en un tipo ideal de la organización), la autoridad y la administración. Los elementos de la burocracia son división del trabajo, jerarquía de autoridad, selección formal, normas y reglamentos formales, impersonalidad en las relaciones y orientación profesional.

Expone Mochón et al. (2014), que también existe el enfoque conductual o del comportamiento organizacional que investiga los comportamientos de las personas en el desempeño de su labor y combina la organización del trabajo, la motivación y las relaciones humanas.

Los principales defensores de esa teoría son Mary Follet (1868-1933), (Mochón,2006), (Doménec, 2007) y a Elton Mayo (1880-1949) (Mayo, 1933). Mary Follet nació en Bostón, fue escritora, trabajadora social y consultora americana, destacó la importancia de los aspectos humanos en la organización, lo cual no lo había tomado en cuenta Taylor; asimismo, propugnó que los trabajadores pueden contribuir positivamente a los resultados de la empresa cuando los gerentes les permitan tomar iniciativas en su labor diaria, debido a que los trabajadores son los que conocen los procesos por lo cual deberían de controlarlos y no los gerentes. 
Propuso que los gerentes deberían ser los facilitadores o coaches más no supervisores o monitores, señalando que deberían motivar a sus empleados y animarlos a que realicen su trabajo con alto rendimiento y comprometidos a lograr los objetivos de la organización.

Elton Mayo, sociólogo y psicólogo australiano, considerado padre del comportamiento organizativo, se enfocó en estudiar al efecto psicológico que tenían las instalaciones de la organización en el trabajador y cómo incidían en la producción, demostró que se debe considerar al trabajador en los proyectos para poder alcanzar los objetivos propuestos.

Por último, el enfoque de contingencias indica la necesidad de adaptar las teorías de la administración y sus métodos a las características de cada organización y a las diferentes situaciones que se enfrentan las organizaciones en función de su entorno y otros factores, entre ellos los municipales. Ahora en esta investigación nos centraremos en los conceptos descritos por diferentes autores sobre la gestión administrativa.

La palabra Gestión Administrativa - Administración, descrito en su libro titulado Diccionario de la Administración Pública según Bacacorzo (1997), cita que es un "conjunto de acciones mediante las cuales el directivo desarrolla sus actividades a través del cumplimiento de las fases del proceso administrativo: planear, organizar, dirigir, coordinar y controlar” (p. 482).

Por su parte Hellriegel, Jackson y Slocum (2009), indican que para que la gestión administrativa sea eficiente, el administrador requiere desarrollar habilidades básicas como el manejo personal, la comunicación y el trabajo en 
equipo, por lo que debe ejecutar eficientemente cuatro funciones básicas que son la planeación, la organización, la dirección y el control.

Define el Diccionario de la Real Academia de la Lengua Española como gestión que es la acción y efecto de gestionar o es la acción y efecto de administrar, y define a la administración que es la acción y efecto de administrar.

Menciona Chiavenato (2001), que administración es el proceso de planear, organizar, dirigir y controlar el uso de los recursos de la organización a fin de alcanzar objetivos con eficacia y eficiencia.

Según Mochón et al. (2014), mencionan que la administración se fundamenta en coordinar y supervisar las actividades realizadas por las organizaciones a fin de lograr que éstas logren sus objetivos de manera eficaz y eficiente. Las funciones básicas de la administración son planear, organizar, dirigir y controlar, las cuales se encaminan a alcanzar los propósitos establecidos por la organización.

Elsa Leuvany, (2020) en la revista Centro Sur, publica el artículo, “Aplicación de la responsabilidad social empresarial en las empresas ecuatorianas en la última década" aquí considera que, debe existir la adopción de responsabilidad social en bien de los colaboradores y familias. Por esta posición es de comprenderse que los empresarios con el concepto de la gestión administrativa, consideren responsabilidad social en la ciudad del Ecuador.

Aidi Jara, (2020) en su artículo "Finanzas Conductuales en el Adulto Mayor de la ciudad de Girardot", señala que es evidente en los adultos mayores, el 
reflejo de que, en la administración o gestión administrativa, del manejo del dinero de esta población, carecen de prácticas concretas para garantizar un mejoramiento en la calidad de vida. Por lo que es de entenderse que la gestión administrativa, no se está aplicando de la manera razonable.

En base a lo anteriormente expuesto por los diferentes autores, se observa que conceptualizan a la gestión administrativa como la administración, lo cual será base para la presente investigación y se analiza si la gestión administrativa de las municipalidades, cuya función es atender a las necesidades de servicios a la sociedad, ha logrado su objetivo de forma eficaz $\mathrm{y}$ eficiente.

Cabe indicar que para este estudio se ha escogido la definición de Mochón et al. (2014) de su libro "Administración. Enfoque por competencias con casos latinoamericanos", debido a que es más actualizada, tiene un enfoque teórico práctico, no es una fuente primaria, sino que ha tomado conceptos y ha tenido como base a distinguidos autores anteriores; y, por lo tanto, se ha tomado como concepción a la gestión administrativa, que tiene cuatro dimensiones, señaladas por este autor, y dejamos de mencionarlas, por la misma naturaleza de este estudio conceptual.

Ahora bien, de estas acepciones aproximadas de conceptualización de la gestión administrativa, como los objetivos del estudio se propone, construirse posibles cambios en su concepción ampliada a las gestiones municipales o de, los distintos gobiernos locales; como es el caso en el Perú donde se les denomina, municipalidades, y por su propia naturaleza, filosofía y propósito de su existencia, como es la de servir al ciudadano; se hace necesario, plantearse nuevos proposiciones de nuevos sistemas de gestión administrativa 
para gobiernos locales, a fin de causar el impacto social de las comunidades ciudadanas y/o usuarios de los servicios que estas instituciones otorgan a través de sus funcionarios quienes de una permanente, capacitación e incentivos laborales de los servidores en todos sus niveles, puedan dar una mayor eficiencia y eficaz servicio administrativo municipal.

\section{Material y métodos:}

El presente estudio descriptivo teórico, se utilizará el método de análisis síntesis, que nos permitirá hacer un análisis de la revisión documental, encontradas y sintetizar la información relevante, en método inductivo deductivo, para luego llegar a generalizaciones que permitan, demostrarnos la importancia del estudio de la conceptualización de la gestión administrativa.

De la revisión teórica, se utilizó el análisis y síntesis, generalización, de las fuentes documentales, dentro de los periodos preferenciales de cinco años, salvo los teóricos clásicos, referenciándose los descriptores y palabras claves para facilidad de ubicación de la información a los lectores, han considerado lectura de artículos, documentos, de revistas de publicación científicas, así como tesis de doctorandos, internacionales y nacionales, así como versiones literarias en idiomas de ingles y español-

Según Hernández et al. (2014), el recolectar los datos involucra elaborar un plan específico de procedimientos que conduzcan a reunir datos con un propósito determinado. 
Según indicó, Arias (2012), la técnica de recolección de datos "se entiende como el procedimiento o forma particular de obtener datos o información" (p.67).

En cuanto a esta definición nuestro método de recolección de información científica será de la revisión documental a través de fuentes principales y secundarias.

\section{Resultados:}

Del estudio de conceptualización de la gestión administrativa, conforme las teorías de los diferentes autores, se obtuvo que conforme lo explica Baca corzo (1997), que viene a ser "conjunto de acciones mediante las cuales el directivo desarrolla sus actividades a través del cumplimiento de las fases del proceso administrativo: planear, organizar, dirigir, coordinar y controlar"; sin embargo con otro enfoque lo dice Mochón et al. (2014), que también existe el enfoque conductual o del comportamiento organizacional que investiga los comportamientos de las personas en el desempeño de su labor y combina la organización del trabajo, la motivación y las relaciones humanas. Asimismo, se toma en cuenta lo explicado por Frederick W. Taylor, por los años (18561915) (Taylor, 1967), quien sostuvo con mucho esmero, el método científico; para establecer la mejor forma de realizar cualquier actividad en la organización y recomendó la especialización y la división del trabajo. Y Weber, sociólogo, filósofo, historiador, jurista alemán, esbozó los principios de la burocracia (teorías de estructuras de autoridad y relaciones basadas en un tipo ideal de la organización), la autoridad y la administración, por lo que se observó, que al sostener Los principales defensores de las teorías Mary Follet (1868-1933), (Mochón,2006), (Doménec, 2007) y a Elton Mayo (18801949) (mayo, 1933). Y es Mary Follet nació en Bostón, fue escritora, trabajadora social y consultora americana, quien destacó la importancia de los aspectos humanos en la organización, lo cual no lo había tomado en cuenta 
Taylor; asimismo, se propugnó que los trabajadores pueden contribuir positivamente a los resultados de la empresa cuando los gerentes les permitan tomar iniciativas en su labor diaria, debido a que los trabajadores son los que conocen los procesos por lo cual deberían de controlarlos y no los gerentes. Es así como entonces se encontró que, de las distintas acepciones, surge la necesidad de que nos conllevan a buscar una mejor conceptualización reforzada, para uniformizar las tan elementales actividades de gestión administrativa.

\section{Referencias:}

María E. Raffino (2020) "Gestión en Administración.

https://concepto.de/gestion-en-administracion/\#ixzz6rIepQzPm.

Chiavenato Idalberto. Introducción a la Teoría General de la Administración. 8va. ed. México: McGraw Hill, 2014. 562 p. ISBN 9786071509802. http://34.201.234.137/bitstream/continental/2757/1/DO_FCE_MI_SI_UC08

\section{0_20162.pdf}

Mochón, F., Mochón, M. \& Sáez, M. (2014). Administración. Enfoque por competencias con casos latinoamericanos (Primera ed.). México D.F., México:

Alfaomega Grupo Editor,

Campos Encalada, S. F., \& Loza Chávez, P. M. (2011). Incidencia de la gestión administrativa de la biblioteca Municipal ¿Pedro Moncayo ¿de la ciudad de Ibarra en Mejora de la calidad de servicios y atención a los usuarios en el año 2011. Propuesta alternativa (Bachelor's thesis). 
Casco, A. D., Garrido, R. G., \& Moran, E. V. (2017). La gestión administrativa en el desarrollo empresarial. Contribuciones a la Economía, (2017-01). http://eumed.net/ce/2017/1/gestion.html.

Rodríguez, D. (2010). Gestión organizacional. Ediciones UC.

Escudero, R. (2011). Diccionario de memoria histórica. Conceptos contra el olvido, La Catara, Madrid.

McKeith, IG, Boeve, BF, Dickson, DW, Halliday, G., Taylor, JP, Weintraub, D., ... y Kosaka, K. (2017). Diagnóstico y manejo de la demencia con cuerpos de Lewy: Cuarto informe de consenso del Consorcio DLB. Neurología, 89 (1), 88-100.

Rahman, MH (2012). La contribución de Henry Fayol y Frederick Winslow Taylor al pensamiento gerencial: una descripción general. Revista $A B C$ de investigación avanzada, 1 (2), 94-103.

Weller, S. (Ed.). (2012). Xilografías expresionistas alemanas. Corporación de mensajería.

Caldwell, JI y Crippen, C. (2015). La filosofía de liderazgo de Mary Parker Follett (1868-1933). The International Journal of ServantLeadership , 11 (1), 187-227.

Mayo, E. (1949). Hawthorne and the western electric company. Public administration: Concepts and cases, 149-158. 
Baca corzo, G. (1999). Comentarios de Ley de Procedimientos Administrativos. Gaceta Jurídica. Lima.

Hellriegel, D., Jackson, S. E., Slocum, J. W., \& Franklin, E. B. (2002). Administración: un enfoque basado en competencias.

Chiavenato, I. (2001). Administración. Proceso Administrativo. Editorial McGraw-Hill, 3.

Caldwell, JI y Crippen, C. (2015). La filosofía de liderazgo de Mary Parker Follett (1868-1933). The International Journal of ServantLeadership , 11 (1), 187-227.

Chiavenato, I., Fortín de Alvarenga, L., Montalvo Gómez, R., Constans de, C. Y. N., Mendieta Díaz, D. K., \& Morales Hernández, A. M. (2019). Introducción a la teoría general de la administración. Una visión integral de la moderna administración de las organizaciones.

Arias, F. G. (2012). El proyecto de investigación. Introducción a la metodología científica. 6ta. Fidias G. Arias Odón.

Fernández, A., del Río, S., López, V., Bawakid, A., del Jesus, MJ, Benítez, JM y Herrera, F. (2014). Big Data con Cloud Computing: información sobre el entorno informático, MapReduce y los marcos de programación. Revisiones interdisciplinarias de Wiley: minería de datos y descubrimiento de conocimientos, 4 (5), 380-409.

Almarashdeh I., Alsmadi M. (2017), How to make them use it? Citizens acceptance of M-government, Applied Computing and Informatics, Volume 
13, Issue 2, 2017, Pages 194-199, ISSN 2210-8327, https://doi.org/10.1016/j.aci.2017.04.001.(http://www.sciencedirect.com/sci ence/article/pii/S2210832716301193)

Abeysiriwardana P. S.R. Kodituwakku,Evaluation of the Use of Web Technology by Government of Sri Lanka to Ensure Food Sec urity for its Citizens, Procedia Food Science, , Volume 6, 2016, Pages 82-91, ISS N 2211601X, https://doi.org/10.1016/j.profoo.2016.02.018.

Allhoff F.y Henschke A. (2018), The Internet of Things: Foundational ethical issues, Internet of Things, Volumes 1-2, 2018, Pages 55-66, ISSN 2542-6605, https://doi.org/10.1016/j.iot.2018.08.005.(http://www.sciencedirect.com/scie nce/article/pii/S2542660518300532)

Elsa Leuvany, (2020) revista Centro Sur, “Aplicación de la responsabilidad social empresarial en las empresas ecuatorianas en la última década".

http://www.centrosureditorial.com/index.php/revista

Aidi Jara, (2020) en su artículo "Finanzas Conductuales en el Adulto Mayor de la ciudad de Girardot"

https://revistas.uniminuto.edu/index.php/Pers/article/view/ 